\title{
Over the counter sale of topical corticosteroids: the need for debate
}

\author{
SAM SHUSTER
}

Not long ago at a conference of consultant dermatologists I presented the case against making hydrocortisone an over the counter (OTC) product. After debate the case against won the day by 34 to 31 votes. A narrow margin, but it was an impressive victory; before the debate all the dermatologists that I questioned had said that licensing hydrocortisone as an OTC product was "probably all right."

Several pharmaceutical companies are keen to introduce $1 \%$ hydrocortisone as a topical product for sale to the public without prescription. To achieve this, approval would have to be obtained for licensing it as an OTC product. Applications have been rejected in the past but the industry-no doubt looking for ways to recover from recent ill considered government attempts to reduce its profitability -is applying considerable pressure to secure approval because market research suggests that the sales potential is large.

The introduction of OTC hydrocortisone would not only affect dermatologists: general practitioners and other physicians would inevitably have to deal with many of the problems that its unlimited use would bring. So here I present the meat of the debate that took place among the dermatologists and leave the general reader to form his or her own opinion.

\section{The case for OTC hydrocortisone}

The case in support of OTC hydrocortisone is a simple one. Minor skin complaints are common, most are transient, and many go unreported. Most, it is alleged, could be treated effectively and safely with $1 \%$ hydrocortisone. Rashes that do not respond to topical hydrocortisone do not matter, either because they will ultimately remit spontaneously or because the patient will eventually report to the family doctor. Self treatment of trivial dermatoses would save time both for the patient and the doctor, and save money.

Two other threads in the case flow in opposite directions. One says that OTC hydrocortisone is a logical extension of self medication; the other that hydrocortisone is largely ineffective, and thus its unsupervised use is of no importance. Many dismiss the possibility that hydrocortisone will cause appreciable side effects.

\section{The case against OTC hydrocortisone}

The case against OTC hydrocortisone is based on both the weakness of the case for it and the disadvantages that its introduction would bring. Hydrocortisone is an active natural substance and it is the hormone that saves each and every one of us from Addisonian crisis. Applied topically to the rat $1 \%$ hydrocortisone

Department of Dermatology, University of Newcastle upon Tyne, Newcastle upon Tyne NE1 4LP

SAM SHUSTER, FRCP, professor of dermatology reduces specific glucocorticoid cytosol receptors in the skin by $70-80 \%$; put on normal human skin it produces vasoconstriction. The effects of topical $1 \%$ hydrocortisone are primarily local, but occasionally systemic absorption occurs. If OTC preparations are licensed and many people are exposed, iatrogenic Cushing's syndrome will be induced, albeit rarely. The very young are particularly at risk because of their high surface area/body mass ratio, as are patients with abnormal skin that allows increased absorption, and those with impaired hepatic metabolism.

Ill effects of topical steroids have been attributed to fluorination of the corticosteroid molecule, but incorrectly so. Fluorination simply increases potency, and for all known glucocorticosteroids potency and toxicity go hand in hand throughout the potency range. It is impossible to dissociate wanted and unwanted effects, and the introducton of new and varied steroid molecules in synthetic corticosteroid preparations has failed to overcome this.

If hydrocortisone is repeatedly used on normal skin thinning, striae, telangectasia (especially on the face), perioral dermatitis, and rosacea may develop. Resistance to infection is reduced and there is an increase in the colonisation of skin by bacteria, fungi, and yeasts. Corticosteroid habituation may also be induced. In this condition the patient begins using topical corticosteroid, usually for a transient and ill defined condition, and then finds that they cannot stop using it. When they do the skin becomes inflamed and scaly. This condition is seen most often in skin disorders affecting the face such as perioral and seborrhoeic dermatitis and rosacea. If the steroid is stopped completely, after a period of worsening that may be severe and last one to two months, the rash usually regresses. This syndrome is usually seen in patients who are using potent synthetic glucocorticosteroids, but it may occur with $1 \%$ hydrocortisone.

Long term indiscriminate use of topical hydrocortisone may, therefore, induce tolerance and the need for ever more potent preparations to prevent recurrence of disease. If OTC hydrocortisone is sanctioned this would undoubtedly be a common problem, as would delay in diagnosis, for steroids would be used inappropriately, for example, to treat undiagnosed scabies, or to treat the itch of melanocytic naevus undergoing malignant transformation. Contact dermatitis may also be expected to deteriorate because immune sensitivity is increased by continued exposure and the regular use of hydrocortisone would make the rash more tolerable thus allowing the patient to continue contact with the offending allergen.

\section{Arguments and counterarguments}

Some argue that since OTC hydrocortisone is already available in the United States and unwanted effects have not been reported they do not exist. But I know of no study to assess the nature and magnitude of the effects of prolonged use of OTC preparations, and until evidence is available from such studies the absence of negative reports is irrelevant. Proponents of OTC hydrocortisone know that if, in a civil action, they were asked by counsel if they could categorically say that repeated application of hydrocortisone would 
not harm the skin they would have to say no. And if they were asked if it would produce unwanted effects they would have to say yes. Thus the crux of the debate is not over whether OTC hydrocortisone will produce unwanted effects but to what extent.

To suggest that the OTC use of hydrocortisone is simply another example of good self medication is, in my view, an abuse of the principle of self help. It is impossible to equate OTC hydrocortisone for "skin disease" with use of aspirin for headache. There are hundreds of different skin diseases and as a rule it is better to withhold treatment until the condition either disappears spontaneously or until a diagnosis is made. To suggest that the public can diagnose its own rashes and select those that will respond to hydrocortisone is extraordinary-it is difficult enough for a well trained dermatologist. The unsupervised use of OTC hydrocortisone would in fact be more analogous to drug abuse, for there will be no clear indication of need, no definition of response, and no estimate of ill effect.

The argument that says that OTC hydrocortisone is safe because it is ineffective is not only untrue but ill conceived. (Even if it was relatively ineffective it would be appalling to use a potent chemical as a placebo.) The proposal to use concentrations of hydrocortisone lower than $1 \%$ makes little sense because it dilutes the biological effect. Furthermore, there is little doubt that once $1 \%$ hydrocortisone was sanctioned marketing of homoeopathic concentrations would follow and, no doubt, OTC sales of more potent corticosteroids and mixed preparations of steroids plus antibiotics and antifungal agents or both.

\section{Misuse by patients}

If OTC hydrocortisone is introduced it will be used on all members of the family regardless of the nature, severity, or site of their skin disease. Its use could not be regulated, and the suggestion that adequate control might be achieved if only small (say $15 \mathrm{~g}$ ) tubes were sold, one at a time and only by pharmacists, is a sad reflection of our ability to confuse private delusions with public realities. It has also been suggested that careful labelling would ensure that patients use the preparations appropriately. But can doctors really be that naive? Imagine the fun the advertising men would have writing a label implying that the hormone could be used with advantage and safety for anything and everything . . . while maintaining sufficient ambiguity to allow future defence against the lady who attempts to sue after prolonged use of hydrocortisone has resulted in unsightly facial telangectasia.

How careful could the public be when tubes of hydrocortisone appear beside the toothpaste? The name of the product can hardly be expected to warn them. Many are aware of the dangers of "cortisone" and so it may safely be predicted that that is the one name that will not appear on packages, which will, no doubt, sport bland safe sounding names such as Cutaids and Dermatreats.

\section{Therapeutic nihilism}

If OTC hydrocortisone is sanctioned what will the effect be on the practice of dermatology? Would we have to agree that generations of clinicians who have spent (and continue to spend) years carefully separating dermatoses into discrete disorders have been wasting their time? In my view today's dermatologists are at last beginning to develop their therapeutic understanding to a level approaching the nosological skills of yesterday's practitioners and it seems madness to bundle up this new knowledge under the bland wrapper of OTC steroid therapy. Finally, there is no evidence that OTC hydrocortisone would divert the weight of trivial skin disease and leave dermatologists free to concentrate on difficult disease. The use of potent corticosteroids has not reduced patient referral. And in the United States OTC hydrocortisone has not resulted in fewer consultations for skin problems.

\section{Conclusion}

The pharmaceutical industry is pushing to introduce hydrocortisone as an OTC preparation (and more powerful steroids, mixed preparations, and homoeopathic preparations are waiting in the wings). Many dermatologists have been canvassed but their views have been solicited before there has been time to consider the arguments for and against its introduction. The issue has not even been raised with the many other doctors who will inevitably be concerned. I believe that the Royal College of General Practitioners should take up the debate on OTC hydrocortisone. Public bodies too should know more about this proposal to introduce a powerful hormone for unlimited and indiscriminate use. It is of unproven benefit and uncertain safety, and government licensing bodies should not approve its introduction.
Should wheat bran be eaten cooked or uncooked? Does phytic acid in uncooked bran interfere with the absorption of calcium and iron?

There is long standing evidence that the absorption of calcium and iron is reduced when the diet contains substantial amounts of wholemeal bread. ${ }^{1}$ This effect is also seen when large amounts (of the order of $40 \mathrm{~g} / \mathrm{d}$ ) of wheat bran are added to the diet, and there are some well documented reports of extreme effects on the calcium status of very high intakes. There is considerable evidence that the presence of phytates, associated with the dietary fibre, is in part responsible for the reduced absorption of calcium and possibly iron and zinc. Bread production and most forms of mild heat processing allow phytases in the foods to degrade some of the phytate and so lessen the potential effects on mineral absorption. ${ }^{2}$ Generally, therefore, a cooked bran preparation may have some advantages in having a reduced phytate content, although there is some evidence that cooking reduces the effectiveness of the bran on colonic function. ${ }^{3}$ It is desirable to ensure adequate mineral intakes in those people whose mineral status may be compromised by the consumption of high fibre diets. - D A T SOUTHGATE, head, nutrition and food quality division, Food Research Institute, Norwich.

1 Royal College of Physicians. Medical aspects of dietary fibre. Oxford: Pitman Medical, 1980:144-61. 2 Erdman JW, Jr. Bioavailability of trace minerals from cereals and legumes. Cereal Chemistry 1981;58:21-6.

3 Wyman JB, Heaton KW, Manning AP, Wicks ACB. The effect on intestinal transit and the feces of raw and cooked bran in different doses. Am $\mathcal{F}$ Clin Nutr 1976;29:1474-9.
A maternity patient, who had given birth to a healthy baby three days before, was diagnosed as having gonococcal septic arthritis of the knee. Is it likely that this infection was a recently acquired genital gonococcal infection, and if so does not the infection ascend into the uterus?

Disseminated gonococcal infection usually presents with joint pain and swelling. Gonococci are the commonest cause of pyogenic arthritis in young adults, ${ }^{1}$ but nowadays arthritis affects less than $1 \%$ of patients with gonorrhoea (compared with $2-5 \%$ before the introduction of antibiotics) and is more common among women than men. ${ }^{2}$ Patients are often asymptomatic at the presumed sites of entry (the genital tract or oropharynx) but gonococci can often be cultured from these areas. The disease may occur in pregnancy (particularly in the third trimester) and pregnancy may be a precipitating factor in gonococcal arthritis because the increased vascularity of the pelvic organs may facilitate haematogenous seeding of the synovium. ${ }^{2}$ Arthritis generally appears three weeks after exposure, with a minimum interval of six days. ${ }^{2}$ The risks of gonorrhoea in pregnancy are known to include premature rupture of the membranes and chorioamnionitis, ${ }^{3}$ but spread to the uterus is unusual. Gonococcal arthritis is treated with penicillin or erythromycin. ${ }^{12}$ -JAMES OWEN DRIFE, senior lecturer in obstetrics and gynaecology, Leicester.

1 Anonymous. Disseminated gonococcal infection. Lancet 1984;i:832-3.

2 Zbella EA, Deppe G, Elrad H. Gonococcal arthritis in pregnancy. Obstet Gynecol Surv 1984; 39:8-12.

3 Robinson A, Dockeray J, Teeling M, Peacock A, Casey EB. Gonococcal arthritis in pregnancy: a report of two cases. Ir J Med Sci 1982;151:313-4. 